\title{
Direct versus indirect molecular diagnosis of fragile X mental retardation in 40 German families at risk
}

\author{
Otto Knobloch, Frauke Pelz, Ursula Wick, David L Nelson, Barbara Zoll
}

\begin{abstract}
In order to test whether the direct molecular diagnostic approach for fragile $X$ mental retardation (Martin-Bell syndrome, MBS) really makes diagnosis of this disease more precise, we evaluated the results of direct diagnosis in $\mathbf{4 0}$ German families at risk together with the results of an earlier study with closely linked flanking markers in the same families. Of 84 men analysed, 43 showed clinical signs. In 39 of these affected men the disease could be confirmed by direct diagnosis. Compared to cytogenetic data, one man was false negative and two were false positive. Two men, whose status could not be determined by means of RFLP data, proved to be normal transmitting males (NTMs). However, the possibility of being an NTM had to be rejected in one case on RFLP data. Fragile $X$ syndrome could be confirmed in 10 of the 13 women with clinical signs. Compared to cytogenetic data there were three cases of false negative results and one of false positive. All 36 obligate carrier women were detected by the direct approach. In addition, 22 women were newly identified as normal transmitting females (NTFs), among them one woman who could not be identified by cytogenetic means or by analysis with closely linked markers.
\end{abstract}

These findings are discussed in view of the relative reliability of the three diagnostic approaches to MBS. Special attention is drawn to the significance of false negative and false positive results in direct diagnosis.

( $\mathcal{f}$ Med Genet 1993;30:193-7)

Institut für

Humangenetik der

Universität,

Gosslerstrasse 12d, 3400 Göttingen,

Germany.

O Knobloch

F Pelz

U Wick

B Zoll

Baylor College of Medicine, One Baylor Plaza, Texas 77030, USA.

D L Nelson

Correspondence to Dr Knobloch.

Received 6 May 1992. Revised version accepted 2 September 1992.
In 1991 the analysis of the molecular structure at Xq27.3 and the discovery of the FMR-1 gene $^{1}$ made a direct molecular approach for diagnosis of the Martin-Bell-syndrome possible. The disease is characterised by a large expansion of a CGG repeat in an exon of the FMR-1 gene in MBS patients and by a more moderate one in normal carriers. In addition, the disease is accompanied by the methylation of a $\mathrm{CpG}$ island functioning as a regulatory element for the FMR-1 gene. Total methylation completely suppresses transcription of the FMR-1 gene.

The methylation status of the $\mathrm{CpG}$ island and the amplification of the CGG repeat can be detected as length variations using molecular probes in this region in Southern hybridis- ation experiments. Nevertheless, in several studies a drawback in application of this method has been shown, as these genomic aberrations not only produce a single enlarged fragment but in some cases also a smear of many very faint bands whose signals may be washed away during blot washing. For putative female carriers these misleading observations can obscure the determination of genotypes.

As identification of carriers is the central task of molecular diagnosis of this disease, we evaluated the results of our direct approach together with the results of an indirect RFLP study in 40 German families at risk for fra(X) mental retardation. We report the results of this combined study and present a strategy for molecular diagnosis of the Martin-Bell syndrome in families at risk.

\section{Materials and methods}

DNA for family analysis was extracted from peripheral blood lymphocytes using a phenol free method reported by Miller et al..$^{2}$ Gene probes for indirect DNA diagnosis were obtained from different sources: RN1A from B Oostra (Rotterdam), VK23B and VK21C from G Sutherland (Adelaide), and U6.2 from $N$ Dahl (Uppsala). The probe pfxa3 was PCR derived from control DNA as a 462 bp fragment according to sequence data from Kremer et al. ${ }^{3}$ We used $1 \mu \mathrm{mol}$ of each of the primers 5'CGTAGCATGCCCCGGATCCCGTGGGAGATGATG-' (SphI) and GCCTAAGCTTCGGCGCTAGCAGGGCTGAAGAGA-3' (HindIII), containing artificial $S p h$ I and HindIII restriction sites at their $5^{\prime}$ ends for subsequent sticky end cloning, 'standard' PCR buffer, $200 \mu \mathrm{mol} d N T P s$, 2.5 units Taq-Polymerase (Amersham), and $1 \mu \mathrm{g}$ genomic DNA in a total volume of $50 \mu \mathrm{l}$. Thirty-five cycles of PCR consisting of $30^{\prime}$ at $94^{\circ} \mathrm{C}, 45^{\prime}$ at $55^{\circ} \mathrm{C}$, and $60^{\prime}$ at $72^{\circ} \mathrm{C}$ were performed in a 'Biomed Thermocycler 60 '. Calculation of the CGG repeat number in controls and family members using this probe in Southern hybridisation experiments was performed by comparison of electrophoretic mobility relative to an $E c o$ RI/HindIII lambda DNA digest. For standard Southern hybridisations $10 \mathrm{mg}$ DNA were digested with the appropriate enzyme, electrophoretically separated in short $(15 \mathrm{~cm})$ or long $(30 \mathrm{~cm}) 1 \%$ agarose gels, and blotted onto nitrocellulose membranes. Mixtures for prehybridisation and hybridisation with probes pE5.1 and pfxa3 contained $60 \mu \mathrm{g}$ placental 
DNA per ml as a substitute for salmon sperm DNA which is generally used for suppression of hybridisation of probes to repetitive sequences in human DNA. Radioactive labelling of probes was carried out by random priming using Amersham's (UK) multiprime kit. Cytogenetic analyses for fragile $\mathrm{X}$ detection were performed in different cytogenetic laboratories.

\section{Results}

Family investigations using the very closely linked and flanking gene probes $\mathrm{RN} 1 \mathrm{~A}$, $\mathrm{VK} 23 \mathrm{~B}, \mathrm{VK} 21 \mathrm{C}$, and $\mathrm{U} 6.2$ in most of the families led to final diagnostic risks for false results of less than $1 \%$. In many cases normal transmitting males and females could be identified with a very high probability, but in other cases identification was less reliable. Thus, we decided to perform additionally direct molecular diagnosis in order to find out whether this really makes family diagnosis of fra $(\mathrm{X})$ mental retardation more precise (fig 1). All families were taken as new requests for family diagnosis and thus kept anonymous in order to

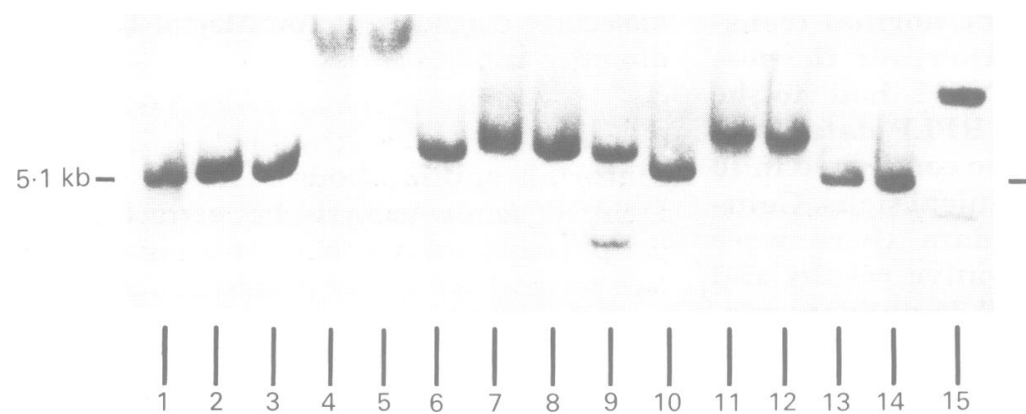

Figure 1 Autoradiograph of a Southern hybridisation of normal controls and MBS patients with probe pE5.1. Autoradiograph shows five controls (lanes 1-3, 13, and patients with probe pene length standard (lane 15), two undigested samples (lanes 4 and 5), and seven proven cytogenetically positive patients (lanes 6-12). Normal band position is at $5.2 \mathrm{~kb}$. Patient in lane 10 has no aberrant fragment and is therefore formally a false negative. Patient in lane 9 shows an additional small extra band beside the larger than normal aberrant band.

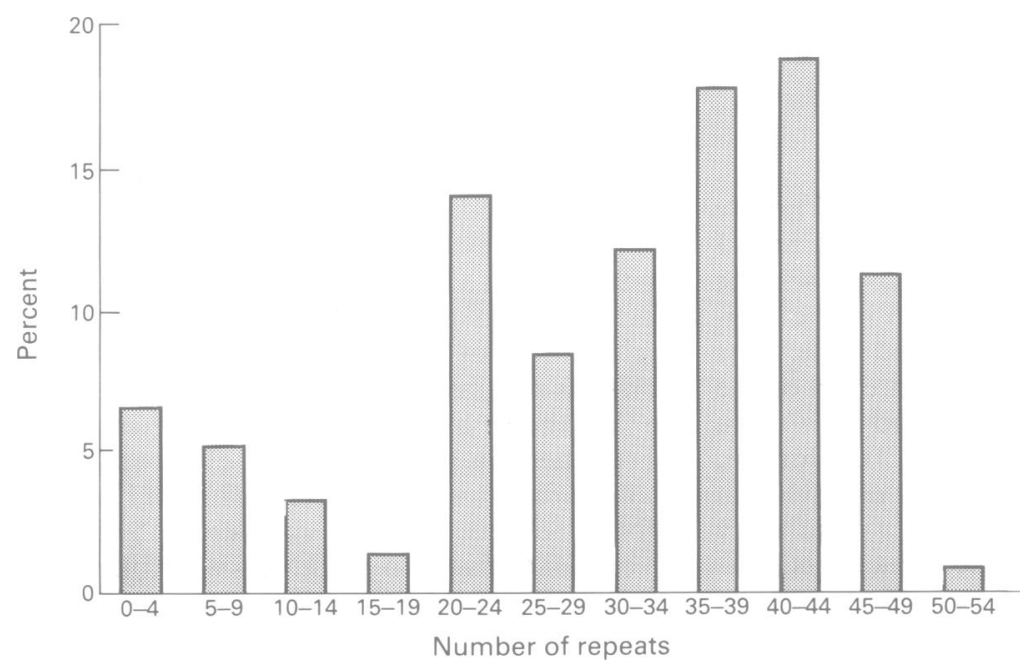

Figure 2 Distribution of CGG repeat length in 213 X chromosomes. DNA of 104 unrelated women and five unrelated men was tested. Southern hybridisation experiments using the probe pfxa3. Length standard for calibrating calculation scale was an EcoRI/HindIII digest of phage lambda. keep the earlier indirect and the planned direct investigation as independent from each other as possible.

First, as the CGG number varies in normal persons too, it was necessary to determine the hybridisation pattern of both probes in normal persons and members of $\mathrm{fra}(\mathrm{X})$ pedigrees. In more than 100 unrelated female controls no aberrant extra bands were seen with probe pE5.1 even on long electrophoretic runs, indicating that normal repeat variation cannot be discriminated by use of this probe (data not shown). Instead, variation of the CGG number was estimated using pfxa3 on Pst I blots (fig 2). All persons subsequently identified as being healthy carriers (NTMs and NTFs, $n=66$ ) had between 54 and 200 CGG repeats. Affected persons of both sexes $(n=56)$ had aberrant bands with at least 260 copies of CGG.

After completion of the direct study, we identified the probands and compared the results of cytogenetic analyses and indirect and direct molecular diagnosis. For this purpose we divided into categories normal transmitting males and females, affected males and females, and normal subjects from $\operatorname{fra}(X)$ families (tables 1 and 2).

All but one of the 26 cytogenetically positive men out of 43 with clinical signs showed aberrations on direct diagnosis (fig 1). Among the 13 women with clinical signs of MBS, nine were cytogenetically positive. Of these women, three showed no aberration. On the other hand there were two cytogenetically negative men who were clearly aberrant, and therefore positive on direct diagnosis. Correspondingly one cytogenetically negative woman was positive by direct diagnosis.

Normal transmitting males and their healthy daughters, normal transmitting females, never or rarely exhibit the fragile site. Eight of the men turned out to be NTMs. As four of them were dead, their NTM status had to be inferred from the band pattern of their daughters and wives. The classification of two of the eight could only be done by means of direct diagnosis, while in the six others direct diagnosis confirmed the RFLP data. A ninth man, already dead too, was originally also regarded as being an NTM by RFLP analysis. This had to be rejected after direct analysis. Thus he accounts for one of the 38 normal men in this study (data not shown). In that group the only remarkable result was one man diagnosed positive by cytogenetic testing but negative on direct diagnosis.

Thirty-six of the 58 women analysed, who were finally categorised as NTFs, were obligate carriers for formal genetic reasons. All these women had aberrant fragments on direct diagnosis.

In order to save DNA for direct diagnosis, RFLP analyses were only completed in 17 of these 36 women. In these cases the results were consistent with the results of the direct analyses.

Of the remaining 22 women, 18 had already been found to be carriers by haplotype analysis. While in a further three of them RFLP 
Table 1 Results of analyses of fra $(X)$ mental retardation in females from 40 families at risk with comparison of cytogenetic, RFLP, and direct molecular data.

\begin{tabular}{lcccc}
\hline & & & \multicolumn{2}{c}{ Direct diagnosis } \\
\cline { 5 - 5 } Probands & Cytogenetic data & RFLP data & Pos & Neg \\
\hline 43 men with & Pos & Y & 24 & 1 \\
clinical signs of & Pos & ND & 1 & 0 \\
MBS & Neg & Y & 11 & 0 \\
& ND & ND & 1 & 0 \\
9 normal & ND & ND & 1 & 0 \\
transmitting males & Neg & Y & $6^{*}$ & 1 \\
(NTMs) & ND & ND & 1 & 0 \\
\hline
\end{tabular}

84 men were analysed. 37 of them proved to be normal in terms of direct molecular diagnosis. Minimum clinical sign was mental retardation to different degrees. Most of the patients showed additional phenotypic involvement including macro-orchidism and distinctive facies. ND = not additional phenotypic involvement including macro-orchidism and distinctive facies. ND = not
determined; RFLP data. Data are in agreement with direct diagnosis: $Y$ (yes), N (no), ND (RFLP notermined; RFLP data. Data are in agreement

* 5 probands were dead; RFLP data were disclosed by family members. In the direct diagnosis * 5 probands were dead; RFLP data were disclosed by family members. In the direct diagnosis
combined fragment aberration in probands' daughters and no aberration in their wives showed their NTM status.

Table 2 Results of analyses of fra $(X)$ mental retardation in females from 40 families at risk with comparison of cytogenetic, RFLP, and direct molecular data.

\begin{tabular}{lcccc} 
& & & \multicolumn{2}{c}{ Direct diagnosis } \\
\cline { 4 - 5 } Probands & Cytogenetic data & RFLP data & Pos & Neg \\
\hline 13 men with & Pos & Y & 6 & 3 \\
clinical signs of & Neg & Y & 1 & 0 \\
MBS & ND & ND & 2 & 0 \\
& ND & Y & 1 & 0 \\
58 normal & Pos & ND & 2 & 0 \\
transmitting & Pos & Y & 14 & 0 \\
females (NTFs) & Neg & ND & 13 & 0 \\
& ND & Uninf & 1 & 0 \\
& ND & & 19 & 0 \\
\hline
\end{tabular}

108 women were analysed. 38 of them proved to be normal in terms of direct molecular diagnosis. Minimum clinical sign of the disease was mental retardation to different degrees. Abbreviations as in table 1 .

* One woman was affected and also had affected daughters. She is accounted for in both categories.
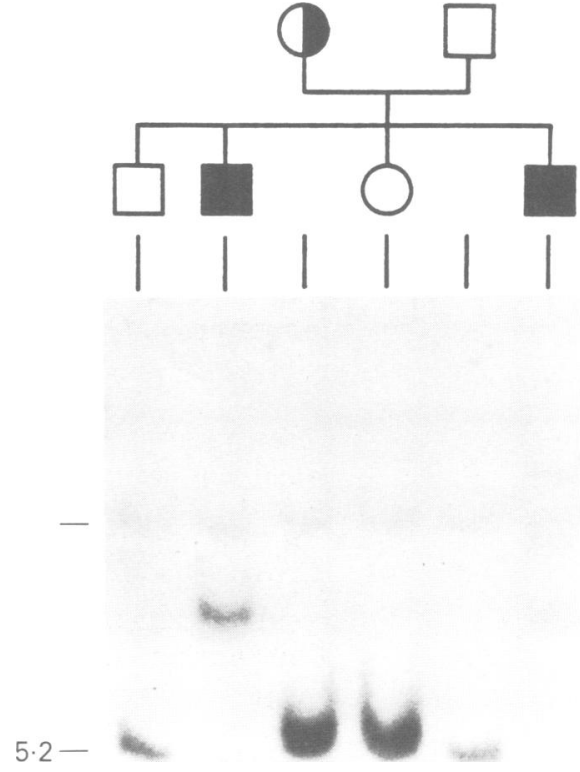

Figure 3 Direct molecular diagnosis in a family at risk for MBS with probe $p E 5.1$. Shaded symbols = affected, half shaded = a carrier. Lane 8 contains a normal control. Normal band position is $5.2 \mathrm{~kb}$, a 'constitutive' band is marked by a sole horizontal bar.

probability. As transmission via the grandmother statistically has only a $6 \%$ probability, it seems more likely that all four daughters have inherited the mutated allele from their normal transmitting (grand)father. In the affected son of one of the carrier women the normal band is absent and instead there is a clearly aberrant one. One of his cousins is healthy and normal in terms of the fra $(X)$ gene, the other affected one lacks the normal band, but he has a different pattern of aberration. His affected sister shows the same aberration on one of her X chromosomes. His healthy sister instead shows a faint smear of bands between the normal band and a constitutive band at roughly $7 \mathrm{~kb}$. The aberrations of both sisters can be seen more clearly in the autoradiograph derived from analysis with the more sensitive probe pfxa3.

As expected, all affected persons in the pedigree have inherited the same $\mathrm{X}$ chromosome from their ancestors, as shown by RFLP data. According to her abnormal band pattern even the healthy girl has inherited this chromosome from her mother too, and in these terms cannot be discriminated from her affected sister. Moreover, this girl is clearly positive on cytogenetic testing. male. The carrier mother of the family and the only sister were heterozygous in terms of hybridisation with $\mathrm{pE} 5.1$, although the bands are not clearly separated from each other and have fused into one thick band. Nevertheless, by this investigation the carriership of the daughter was confirmed.

Another example of direct diagnosis with pE5.1 is shown in fig 4. Four healthy sisters show an additional aberrant fragment at roughly 5.3 to $5.4 \mathrm{~kb}$ beside their normal band. They are clear NTFs. As the grandparents are dead, the question of whether the disease had been transmitted via the grandmother or grandfather can only be answered in terms of

\section{Discussion}

Owing to the unusual mode of inheritance of MBS there are four classes of mutation carriers: affected males and females and normal transmitting males and females. It is generally accepted that fragile $\mathrm{X}$ mental retardation is the result of a two step mutation. The first mutation, called the premutation, leads to a moderate amplification of the CGG repetitive sequence in the FMR-1 gene in a healthy man, who is a normal transmitting male. He passes the premutation without change to all his 


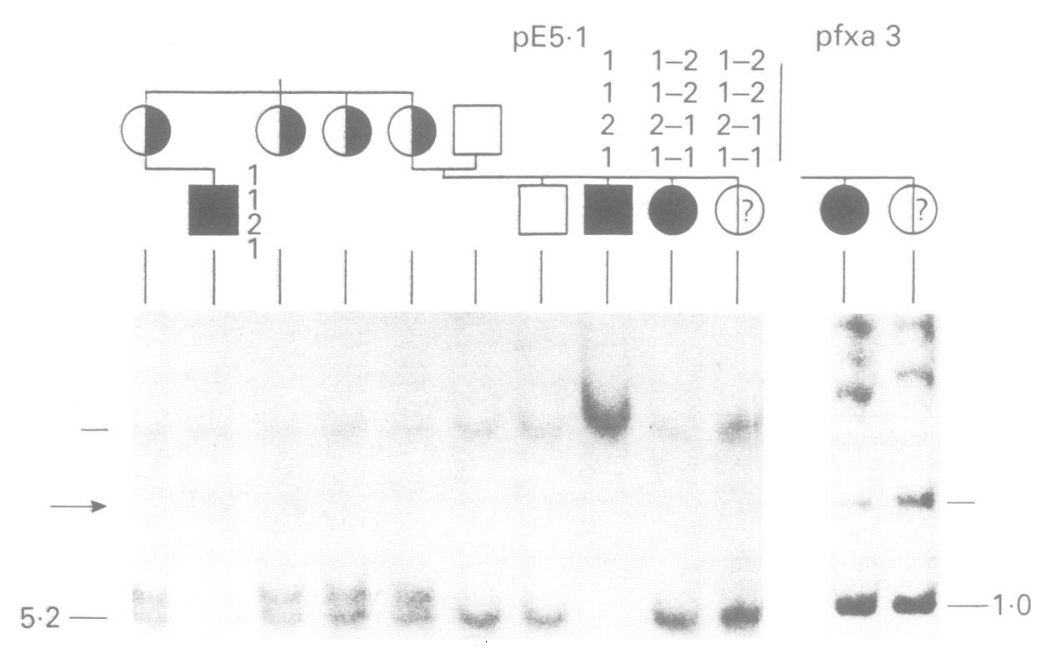

Figure 4 Direct molecular diagnosis in a family at risk for $M B S$ with probes $p E 5.1$ and pfxa3. Symbols as in fig 3. Some allele distributions from parallel RFLP investigations with the probes (from the top to the bottom) VK21C, U6.2, VK23B (XmnI), VK23B (HindIII) are shown. The sisters on the far right are also shown with their pfxa3 autoradiograph. Normal band position is at $5 \cdot 2 \mathrm{~kb}$ for pE5.1 and $1.0 \mathrm{~kb}$ for pfxa3. A 'constitutive' band is marked by a sole horizontal bar. The arrow points to the aberrant band of the patient in lane 2.

daughters. These daughters are healthy, but obligate carriers, so called normal transmitting females. The premutation creates a predisposition for further amplification of the CGG repeat during oogenesis.

This further amplification of the CGG repeat is the second mutational step and is called the full mutation. As the term predisposition suggests, the full mutation does not take place in all premutated oocytes but in most of them. As a result, $80 \%$ of grandsons and $30 \%$ to $50 \%$ of granddaughters who inherit the abnormal $X$ chromosome develop the full disease, whereas $20 \%$ of the grandsons and $50 \%$ to $70 \%$ of the granddaughters with the mutated $\mathrm{X}$ chromosome are healthy but again normal transmitting carriers. This makes prenatal diagnosis difficult, especially because only a positive cytogenetic test is reliable but not a negative one. Under these preconditions, a method for the diagnosis of MBS must fulfil four requirements: (1) diagnosis of the disease, (2) clear identification of carriers of both sexes, (3) reliable prenatal diagnosis, (4) predictive discrimination of carriers of both sexes with and without symptoms of the disease prenatally or later on before puberty.

While reliability of the cytogenetic tests is limited and RFLP analyses, apart from the possibility of total uninformativity, ignore the special problems in prenatal diagnosis of fra (X), the direct approach promises to overcome most of these difficulties. As for prenatal diagnosis there still remains some uncertainty in interpreting direct results, the direct approach most readily fulfils requirements (1) and (2). We not only found all obligate carrier women aberrant on direct molecular diagnosis but also identified 22 other women as carriers, of whom only five had been identified by cytogenetic means and of whom one could not be identified as a carrier by RFLP investigations owing to uninformativity. These results are in good agreement with those of other research groups, although two problems have to be faced in identification of female carriers. The first is the possible degeneration of the aberrant band to many very faint bands (fig 4), which might be washed away during blot washing procedures. This can happen in male patients too, especially with probe pfxa3, but in contrast to women there remains no normal band from the second $\mathrm{X}$ chromosome, which can lead to misclassification of the woman as homozygous normal (fig 5).

The second problem is the fact that the CGG repeat number varies even in normal persons, with an average copy number of $29 .{ }^{4}$ The repeat becomes unstable within a range from 46 to 54 repeats. Because normal subjects show a range in allele size varying from a low of six to a high of 54 repeats, and premutations range in size from 52 to over 200 repeats, ${ }^{4}$ there is a small overlap of the normal and premutated size area.

An additional problem concerning carrier women is shown in fig 4 . We had shown with closely linked flanking markers that both daughters inherited the same $\mathrm{X}$ chromosome. One of the daughters was affected and the other healthy but cytogenetically positive and showing even more aberrant fragments on direct diagnosis than her affected sister.

We think that this case is an example of extreme Lyonisation. On this assumption, the healthy girl bears the full mutated $\mathrm{X}$ chromosome which was preferentially inactivated in such a high proportion of cells that even the dominant character of the mutation was overruled by the large majority of normal X chromosomes.

Despite these drawbacks, carrier diagnosis using the direct approach seems to be very reliable as the problematical cases described above are very rare. Larger numbers of women investigated will make it possible to confirm a precise risk figure for false results in direct carrier diagnosis. The application of PCR is already established for amplification of CGG repeats in normal persons, normal transmitting males, and carrier women with low aberrant copy numbers ${ }^{5}$ and is capable of delivering more precise data on CGG copy number, but cannot overcome the biological phenomenon of overlapping ranges in normal women and carriers. It may be even more complicated if the DNA of a woman with severely degenerated aberrant bands is amplified.

Diagnosis of the disease itself in affected persons has a high reliability, although we found one man to be clearly false negative compared to cytogenetic analysis. In addition, of those healthy normal persons who were formerly cytogenetically tested positive during a search for carriership, one man and two women were found to be normal on direct diagnosis. Nakahori et $a l^{6}$ also had three cases of false negative results in patients and Suthers et al had one (Poustka, personal communication), but both groups explained this discre- 


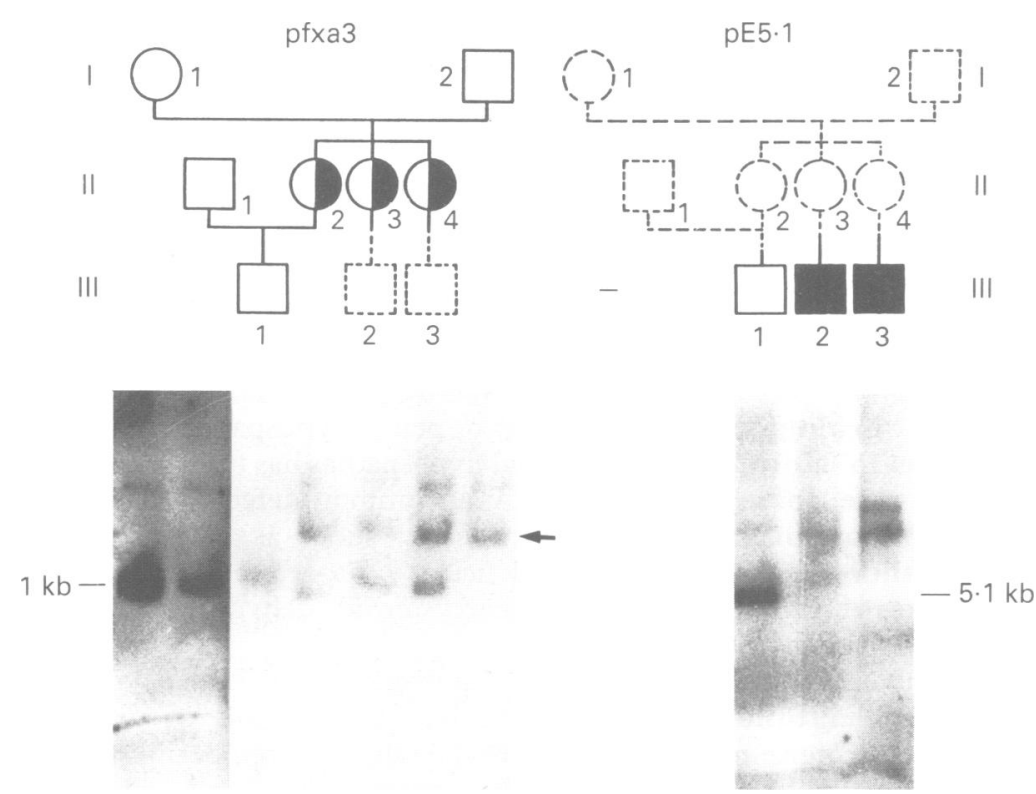

Figure 5 Direct molecular diagnosis in a family at risk for $M B S$ with probes pfxa3 and pE51.1. Symbols as in fig 3. The arrow marks the aberrant band. On the left (with probe pfxa3) affected persons $I I I \cdot 2$ and III.3 are omitted, as their aberrant bands were invisible. With probe $p E 5.1$ degeneration was less and aberrant bands could be seen. This is shown on the right of the figure.

pancy as resulting from fragility at a locus close to and indistinguishable from Xq27.3.

Thus it seems as if discrepancies between cytogenetic data and direct molecular diagnosis are not negligible. Consequently, these affected men found to be false negatives and the directly diagnosed patients are suffering from different diseases which are clinically indistinguishable. This different $\mathrm{X}$ linked hereditary form of mental retardation has nothing to do with a lack of or diminished production of the FMR-1 gene product as was shown in true MBS patients by Pieretti et al. ${ }^{8}$

The other way of comparing cytogenetic and direct molecular data was shown by finding two cytogenetically negative men with MBS 'false positive' on the direct approach. One explanation for this phenomenon was proposed by Pergolizzi et $a l^{9}$ who assumed that the expression of a fragile site might be correlated with the extent of CGG amplification: affected men may possibly be negative on cytogenetic testing, if their CGG repeat remains comparatively small. Considering that the rate of misdiagnosis in the cytogenetic test is about $5 \%$ owing to rare false positive and more frequent false negative diagnoses, ${ }^{10}$ we think that the observed phenomenon is because of the lesser reliability of the cytogenetic test. Thus, direct molecular diagnosis offers an excellent means for differentially diagnosing mentally retarded persons for fra $(X)$ mental retardation even if they do not exhibit the fragile site.

A strategy for the diagnosis of $\mathrm{fra}(\mathrm{X})$ mental retardation which considers the possible prob- lems of carrier detection should follow this procedure: (1) Southern hybridisation of EcoRI digested DNA to the direct probe pE5.1. Every woman found to be heterozygous even on long electrophoretic runs is a carrier of MBS. (2) Women or men with no aberrations with pE5.1 but strongly suspected of being carriers should additionally be analysed with the more difficult to handle but more sensitive probe pfxa3 on PstI blots. (3) To determine the CGG copy number in the most critical persons the PCR protocol of Fu et al ${ }^{4}$ can be followed. (4) To confirm the results, RFLP analysis or analysis with microsatellites as markers of the affected chromosome can be performed. ${ }^{17}$

The correlation between the copy number and the severity of the disease is a good means for prenatal diagnosis. If there are small inserts detected in most cases this should account for healthy carrier fetuses. Whether methylation status of the $\mathrm{CpG}$ island at $\mathrm{Xq27.3}$ can give additional information is not clear as there are contrary reports on the reliability of results for methylation status in villi. While Sutherland et $a l^{10}$ showed that methylation status in villi does not reflect that of the embryonic tissue, Oberle et $a l^{11}$ found it very useful to determine the methylation status in villi. Further investigations will give information about the usefulness of the methylation status in chorionic villi for prediction of the severity of fragile $\mathrm{X}$ mental retardation.

We are grateful to $M$ Schloesser and V Bochdalofsky for criticising the manuscript, I Reise for secretarial help, and C Gilat for photographic assistance. We thank all the families who provided us with blood samples.

1 Verkerk AJMH, Pieretti M, Sutcliffe JS, et al. Identification of a gene (FMR-1) containing a CGG repeat coincident with a breakpoint cluster region exhibiting length variation in fragile X syndrome. Cell 1991;65:905-14.

2 Miller SA, Dykes DD, Polesky HF. A simple salting out procedure for extracting DNA from human nucleated cells. Nucleic Acids Res 1988;16:1215-6.

3 Kremer EJ, Pritchard M, Lynch M, et al. Mapping of DNA instability at the fragile $X$ to a trinucleotide repeat sequence p (CCG)n. Science 1991;252:1711-4.

4 Fu Y, Kuhl DPA, Pizzuti A, et al. Variation of the CGG repeat at the fragile site results in genetic instability:

5 Yu S, Pritchard M, Kremer E, et al. Fragile X genotype characterized by an unstable region of DNA. Science
chat 1991;252:1179-81.

6 Nakahori Y, Knight SJL, Holland J, et al. Molecular heterogeneity of the fragile $\mathrm{X}$ syndrome. Nucleic Acids Res 1991;19:4355-9.

7 Suthers GK, Mulley JC, Voelckel MA, et al. Linkage homogeneity near the fragile $X$ locus in normal and fagile $X$ families. Genomics 1991;10:576-82.

8 Pieretti M, Zhang F, Fu Y, et al. Absence of expression of the FMR-1 gene in fragile X syndrome. Cell 1991;66:81722.

9 Pergolizzi RG, Erster SH, Goonewardena P, Brown WT. Detection of full fragile $X$ mutation. Lancet 1992;339:271-2.

10 Sutherland GR, Gedeon A, Kornman L, et al. Prenatal diagnosis of fragile X syndrome by direct detection of the
unstable DNA sequence. $N$ Engl f Med 1991;325:1720-2.

11 Oberlé I, Rousseau F, Heitz D, et al. Instability of a 550 fragile X syndrome. Science 1991;252:1097-102. 